\title{
Electrical impedance myography for assessing paraspinal muscles of patients with low back pain
}

\author{
Yun Wang, $\mathrm{MD}^{1,2}$, Laura Freedman, $\mathrm{NP}^{3}$, Martin Buck, $\mathrm{BS}^{3}$, Jose Bohorquez, $\mathrm{PhD}^{3}$, Seward B. Rutkove, $\mathrm{MD}^{2,5}$, \\ and John Keel, MD
}

1. Department of Neurology, Beijing Chaoyang Hospital, Capital Medical University Beijing, China.

2. Department of Neurology, Beth Israel Deaconess Medical Center, Harvard Medical School, Boston, USA.

3. Myolex, Inc, Brookline, USA

4. Emory Orthopaedics/Spine Center, Emory University, Atlanta, USA

5. Email any correspondence to: srutkove@bidmc.harvard.edu

\begin{abstract}
The objective of this study was to determine the potential value of electrical impedance myography (EIM) for assessing lumbosacral paraspinal muscle (LPM) condition in lower back pain (LBP) patients. Standard methods for assessing the condition of LPMs, such as magnetic resonance imaging, are inconvenient and expensive. One tool that could be useful for this purpose is electrical impedance myography (EIM) a technique that can be performed rapidly at the bedside. After undergoing a screening history and examination, subjects were studied with the mView EIM device (Myolex, Inc, Boston). Bilateral LPMs were measured three times each and the two closest sets of measurements averaged on each side. Data analysis included non-parametric twogroup comparisons between healthy subjects and back pain patients, receiver-operating curve analyses, and correlation analyses to age and body mass index. A total of 86 healthy individuals (median age (interquartile range) (IQR), 45.5 years (30.3-56.0 years), 42 men, 44 women) and 47 LBP (median age 51.0 year (39.5-57.5) years, 21 men, 26 women) were enrolled. Median EIM $100 \mathrm{kHz}$ phase was lower in the LBP patients $\left(9.3^{\circ}\left(\right.\right.$ IQR8. $4^{\circ}$ $10.6^{\circ}$ ) versus $\left.11.4^{\circ}\left(\operatorname{IQR} 9.4^{\circ}-13.0^{\circ}\right), \mathrm{p}=0.0007\right)$. Significantly increased normalized side-to-side differences were present for all three EIM variables (e.g., median $100 \mathrm{kHz}$ phase 0.15 (IQR 0.07-0.31 in LBP patients versus 0.09 (IQR 0.04-0.17) in healthy individuals). A significant correlation between $100 \mathrm{kHz}$ EIM phase and reactance was found with age $\left(R_{\text {spearman }}=-0.46, P=0.0002\right.$ and $R_{\text {spearman }}=-0.440$, $\mathrm{P}=0.0003$ ) but not for resistance. This study provides early evidence supporting that EIM has the potential to serve as a useful tool for evaluating the condition of LPMS.
\end{abstract}

Keywords: Low back pain; paraspinal muscles; electrical impedance; radiculopathy; musculoskeletal; bioimpedance; asymmetry; age; body mass index

\section{Introduction}

Low back pain (LBP) can originate from a variety of conditions, ranging from radiculopathy to facet joint arthritis to simple deconditioning [1-3]. While the pathologies that underlies LBP can be quite variable, a common theme for therapy focuses on the improvement in the condition and strength of the lumbar paraspinal muscles (LPMs), including the erector spinae and multifidus muscles, as well as abdominal muscles [4-8].

Despite the importance of lower back muscle condition, there exist few good techniques for assessing muscle condition of the LPMs. While surface electromyography can provide insight into the activity of those muscles, it does not provide a readily quantifiable index of muscle condition [9]. Radiological techniques, including computerized tomography and magnetic resonance imaging, have been used for this purpose and can provide information on muscle fat infiltration and size [10-13]. Yet, to date, an easily applied device that can be employed at the bedside for assessment of lower back muscles by physicians or by physical therapists does not exist.

One technology that holds promise in this regard is electrical impedance myography (EIM). EIM is an electrical 
bioimpedance based technique in which the current emitting and voltage sensing electrodes are placed in close proximity to one another on the skin overlying a muscle of interest [14]. The technique has been primarily used in the assessment of a variety of neuromuscular conditions, including amyotrophic lateral sclerosis, muscular dystrophy, and localized nerve injury [15-17]. However, the technique is sensitive to subtler muscle abnormalities, including simple disuse atrophy due to immobility or injury [18]. To date, the technique has only been applied in a single study of the LPMs in older adults with comparison to computerized tomographic imaging [19].

In this study, we sought to further investigate EIM for the assessment of a group of patients with LBP due to a variety of etiologies as compared to a group of healthy participants without significant lower back pain. Our hypotheses were: 1. That LBP patients would, on average, demonstrate abnormal paraspinal muscle impedance values as compared to the heathy participants and 2 . That patients with LBP would demonstrate greater side-to-side difference in impedance values than the healthy controls, suggesting underlying asymmetries in muscle condition.

\section{Materials and methods \\ Low back pain patients and healthy participants.}

The study took place at New England Baptist Hospital, Boston, MA and Myolex, Inc (previously Skulpt, Inc), Boston, MA. Potential participants who passed all the inclusion and exclusion criteria were enrolled in the study. Patient inclusion criteria included: Ages 21-80 years, lower back pain attributable to a spinal etiology as determined by boardcertified physiatrist (J.K.). Exclusion criteria included, history of a generalized neuromuscular condition, except for mild polyneuropathy or common mononeuropathies (e.g., carpal tunnel syndrome, ulnar neuropathy at the elbow), history of moderate-to-severe ongoing medical conditions producing generalized disability, such as advanced cardiac or renal disease, metal spine implants of any type. For healthy subjects, the exclusion criteria included the above items as well as a history of present or past disabling back or neck pain or a history of sciatica or cervical radiculopathy (the cervical spine was also studied, but is not included in this analysis, given substantially smaller number of patients with this problem who were ultimately recruited). In addition to disease/ health category, the participants' sex, age, height, and weight were also recorded.

\section{Informed consent}

Informed consent has been obtained from all individuals included in this study.

\section{Ethical approval}

The research related to human use has been complied with all relevant national regulations, institutional policies and in accordance with the tenets of the Helsinki Declaration, and has been approved by the authors' institutional review board or equivalent committee.

\section{EIM data collection}

The Myolex ${ }^{\circledR}$ mView $^{\mathrm{TM}}$ device was used for this data collection. Briefly, this device consists of a small laptop computer attached to power convertor and an impedance measuring unit configured with a disposable electrode array (see Figure $1 \mathrm{~A}$ ) that performs standard 4-electrode impedance measurements. It collects 41 frequencies of data from $1 \mathrm{kHz}$ to $10 \mathrm{MHz}$ using 3 different electrode configurations (see Figure 1B), although for the subsequent data analysis, only the configuration with the greatest muscle depth penetration was used ("configuration 1" with the electrodes spaced furthest apart). An example of typical multifrequency data obtained from the $\mathrm{mView}^{\mathrm{TM}}$ is shown in Figure 2 below.
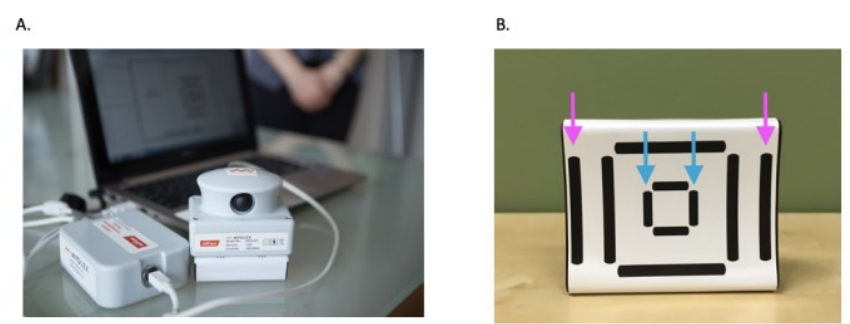

Figure 1. A. The Myolex $\mathrm{mView}^{\mathrm{TM}}$ system for neuromuscular assessment, including laptop, power convertor box, impedance measuring unit, and electrode array; B. Detail of electrode array used in this study; magenta-current emitting, blue-voltage sensing. Only data from these 4 electrodes were used in this analysis.

The software on the laptop allows the muscle of interest to be chosen and provides basic instructions for the application of the device to the participant. The patient is placed in a standard position (seated, leaning forward) and the skin over the L4-5 paraspinal regions moistened with saline. The electrode array is then applied to the skin on one side of the spine, and a measurement is taken (in about 5 seconds). The electrode array is then lifted, the skin remoistened, the electrode array applied again, and the measurement repeated; this procedure is then completed a third time. An average of the two closest readings is then calculated. The examiner then performs the measurement on the contralateral side.

\section{Data analysis}

The main data of interest in this study was the $100 \mathrm{kHz}$ phase, reactance, and resistance values (a standard trio of impedance measures) using the widest electrode configuration.

This $100 \mathrm{kHz}$ frequency value is less contaminated by contact artifact (due to inconsistent contact between the 
electrode and the skin) than the $50 \mathrm{kHz}$ phase value, which has also been commonly used [15]. Thus, while a number of potential analyses are possible for the purposes of this study we chose this one simple set of 3 measures. Data was analyzed using Prism 8.0 (Graphpad, Inc). Median and interquartile range (IQR) were reported for all measures; standard two-group comparisons were completed using Mann-Whitney $U$ test. Receiver operating characteristic (ROC) curves were also generated to determine the capability of the EIM measures alone to distinguish LBP patients from healthy controls [20].

Correlation analyses were completed using Spearman rank correlation. For all analyses, significance was accepted at $p<0.05$, two-tailed.

\section{Results}

\section{Demographic data}

A total of 86 healthy individuals (median age (interquartile range) (IQR), 45.5 years (30.3-56.0 years), 42 men, 44 women) and 47 low back pain patients (median age 51.0 years (39.5-57.5) years, 21 men, 26 women) were studied.

The difference in age was not significant $(p=0.11)$ ). These data, as well as weight and height, are summarized in Table 1.

Table 1. Demographic data

\begin{tabular}{|l|c|c|}
\hline & $\begin{array}{l}\text { Low Back Pain } \\
\text { Participants }\end{array}$ & Healthy Volunteer \\
\hline Participants & 47 & 86 \\
\hline Median age, IQR & $51.0(39.5-57.5)$ & $45.5(30.3-56.0)$ \\
\hline Sex (male/female) & $21 / 26$ & $42 / 44$ \\
\hline Body weight (lbs) & $165.0(147.0-187.5)$ & $162.0(136.3-197.0)$ \\
\hline Height (inches) & $67.0(64.5-70.0)$ & $66.0(64.0-69.0)$ \\
\hline
\end{tabular}

\section{EIM outcomes}

The raw median EIM $100 \mathrm{kHz}$ phase, averaged across both sides, was lower in the LBP patients $\left(9.3^{\circ}\right.$ (IQR $8.4^{\circ}-10.6^{\circ}$ ) versus $\left.11.4^{\circ}\left(\operatorname{IQR} 9.4^{\circ}-13.0^{\circ}\right), \mathrm{p}=0.0007\right)$, although the raw resistance and reactance values were not different between the groups (Figures $3 A, B, C$ ). To determine the potential for these single measures could discriminate effectively between the two groups of individuals, ROC analyses were also constructed (Figures D, E, F); while resistance and reactance showed values no better than chance, the phase values did have an area under the curve (accuracy) of $61 \%$.

In addition to evaluating the raw impedance data, we also analyzed the side-to-side differences to obtain a measure of asymmetry, again anticipating that LBP patients would have greater asymmetry. This was calculated by the taking the difference in each impedance parameter between the two sides and dividing by the mean value of the two sides. Significantly increased normalized side-to-side differences were present for all three EIM variables (e.g., median $100 \mathrm{kHz}$ phase 0.15 (IQR 0.07-0.31) in LBP patients versus 0.09 (IQR 0.04-0.17) in healthy individuals), as shown in Figures 3A, B, C). These asymmetries showed a somewhat better ability to discriminate between LBP patients and healthy subjects than the raw impedance values, with the area under the curve being above 0.60 for all 3 measures (Figures D, E, F).

\section{Correlation analyses}

Significant correlations of $100 \mathrm{kHz}$ EIM phase and reactance with age were identified, $\left(R_{\text {spearman }}=-0.46, P<0.0001\right.$ and $R_{\text {spearman }}=-0.440, P=0.0001$ ) but not for resistance (Figure $5 \mathrm{~A}$, $B, C)$. Weaker correlations were found between body mass index (BMI), calculated as body mass $/$ height $^{2}$, and the EIM values, although both phase and resistance values were significant $\left(R_{\text {spearman }}=-0.39, P=0.0009\right.$ and $R_{\text {spearman }}=-0.31$, $\mathrm{P}=0.008$, respectively). See Figure $5 \mathrm{D}, \mathrm{E}, \mathrm{F}$.

\section{Discussion}

This study provides early evidence that EIM values may be helpful in the evaluation and therapy of patients with LBP, demonstrating group differences between those with pain and those without. Ultimately, the real values of the technology will be in determining whether such EIM measures may be helpful in serving as tools to assist in tracking the effects of treatment, including LPM strengthening, with results being gauged by using EIM as an outcome measure. While no longitudinal analysis was
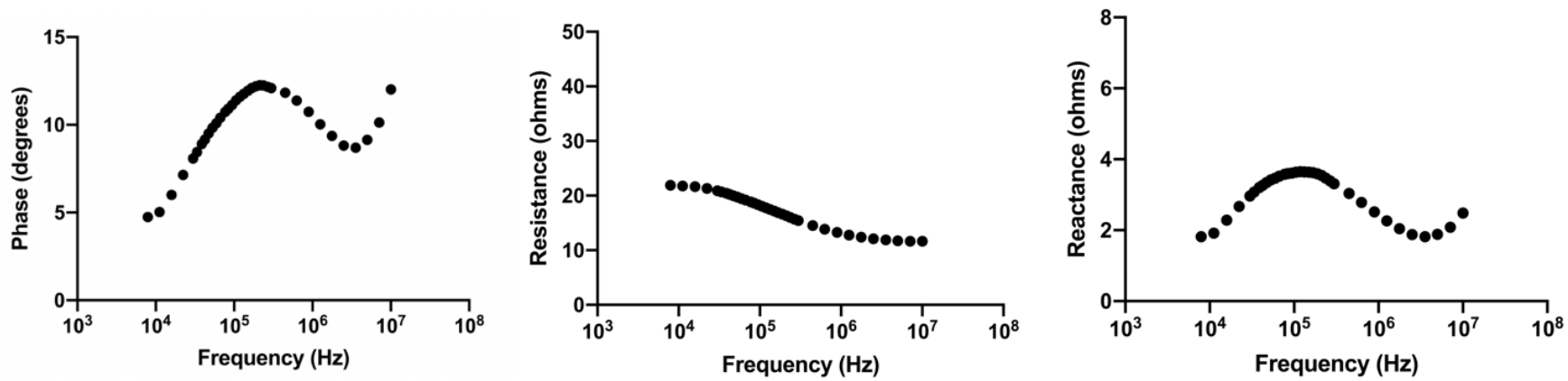

Figure 2. Typical multifrequency data obtained from with the mView impedance-measuring system shown in Figure 1. This data is from a 34 -yearold healthy volunteer. 
Phase

$\mathrm{P}=0.0007$

A.
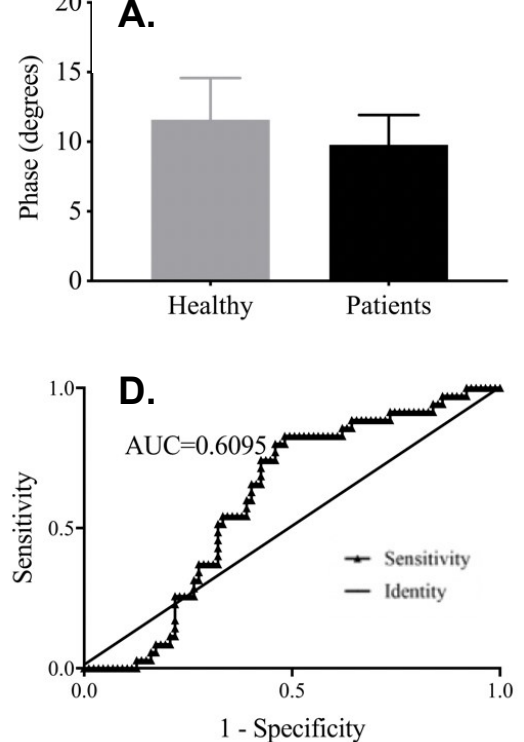

Resistance

$\mathrm{P}=0.131$
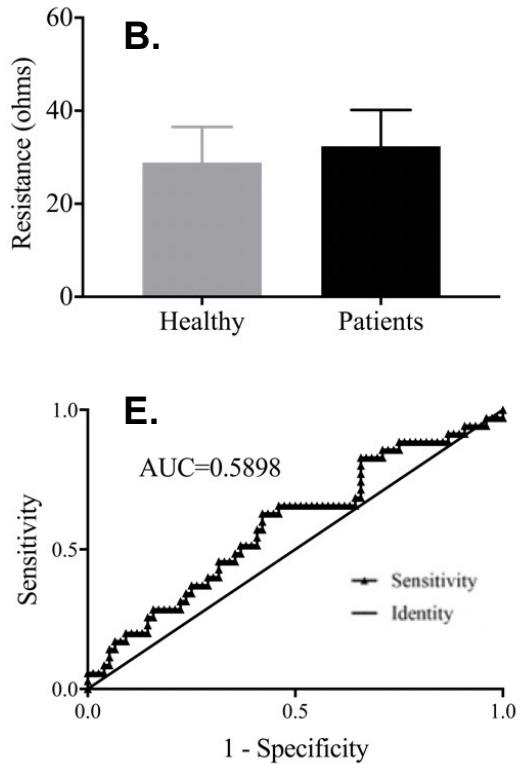

Reactance

$\mathrm{P}=0.127$
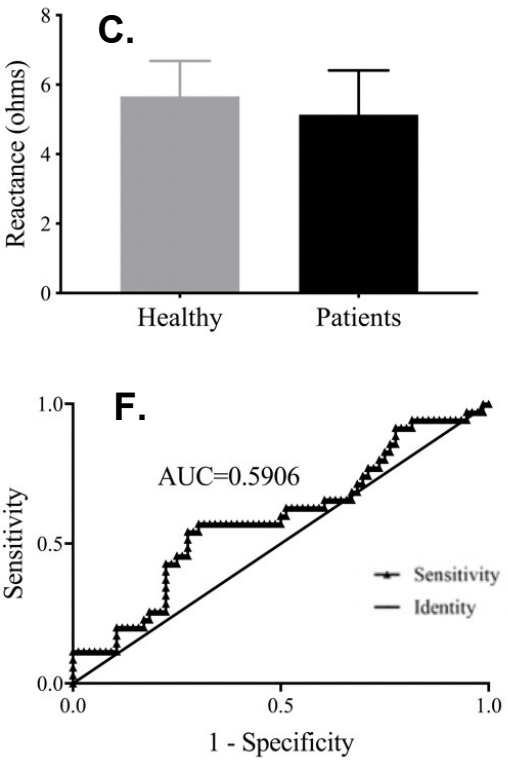

Figure 3. A, B, C. The phase, resistance, and reactance values at $100 \mathrm{kHz}$ ( \pm standard deviation) for low back pain patients versus healthy controls. $\mathbf{D}, \mathbf{E}, \mathbf{F}$. Receiver operating characteristic (ROC) plots for these same values. While the population means may be different, this analytical approach has limited value in discriminating between LBP patients and healthy individuals.

Phase

$\mathrm{P}=0.013$

A.
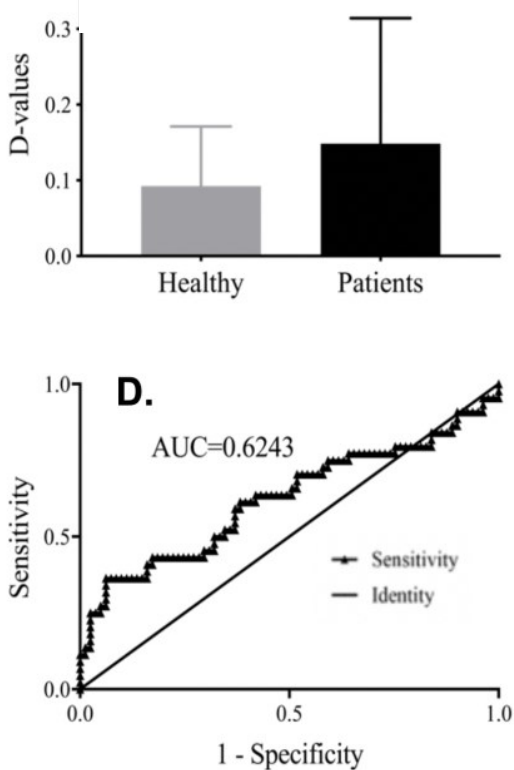

Resistance

$\mathrm{P}=0.003$
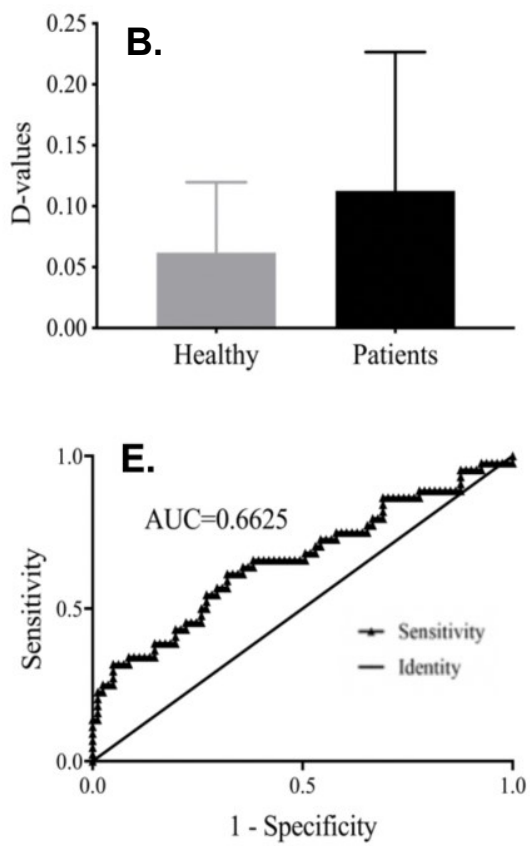

Reactance

$\mathrm{P}=0.012$
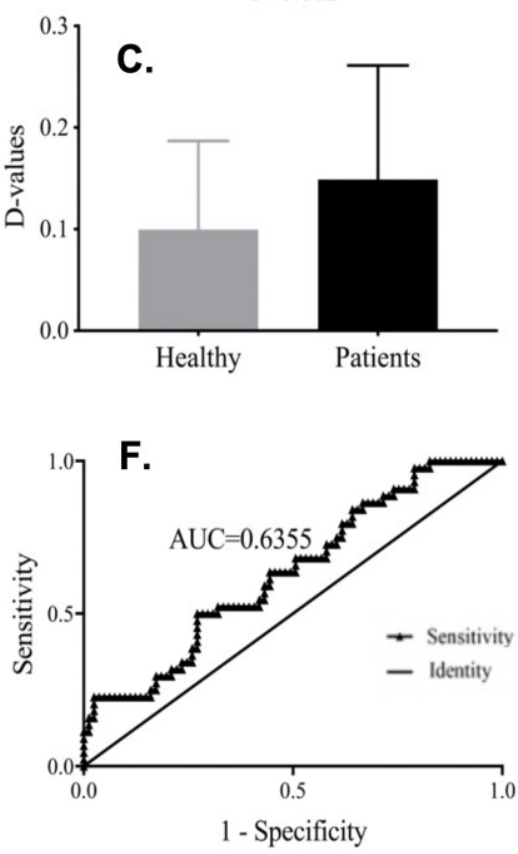

Figure 4. A, B, C. Relative differences in $100 \mathrm{kHz}$ values (absolute value (right-left difference /right-left average)) for low back pain patients versus healthy controls. D, E, F. ROC plots for these same values. The D-value is equal to the side-to-side difference divided by the average of both sides. While still not a strong discriminator, this approach is stronger.

completed here, this study provides initial proof-of-principle that such EIM-based measures may be useful to track.

The three main impedance variables, phase, resistance, and reactance all showed their own unique behaviors in this analysis. As is observed in most neuromuscular disorders, the two primary impedance values, resistance and reactance, moved in opposite directions, with resistance increasing and reactance decreasing, albeit insignificantly (Figures $3 \mathrm{~B}, \mathrm{C}$ ). But these differences led to an overall reduction in phase (calculated as phase $=$ arctan (reactance) resistance). The asymmetries are also in keeping with our initial hypotheses, including the fact that patients with LBP, regardless of etiology, tend to have greater asymmetry in all 3 impedance measures. 


\section{Correlations to age}
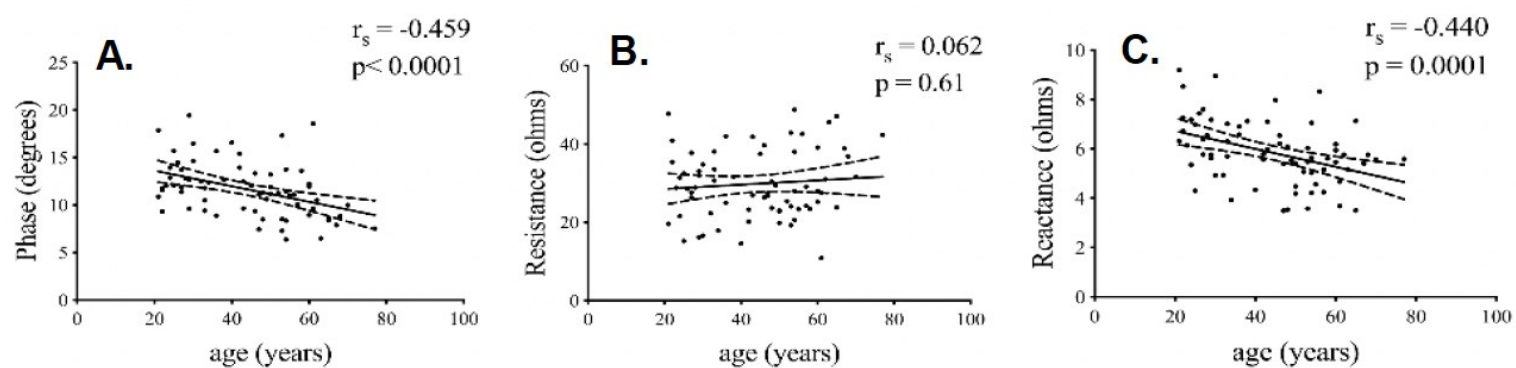

\section{Correlations to $\mathrm{BMI}$}
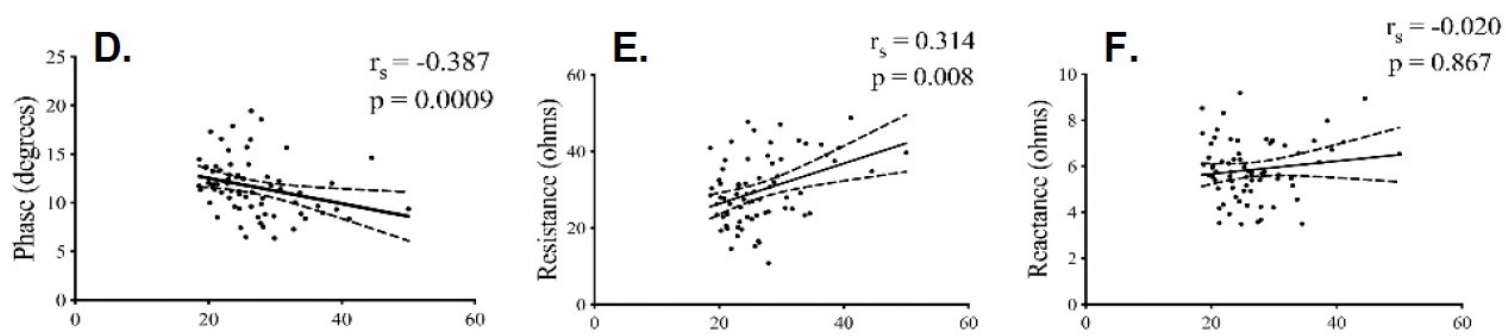

Figure 5. A, B, C. Correlation between phase, resistance, and reactance values at $100 \mathrm{kHz}$ and age. Both phase and reactance show a significant relationship, whereas resistance does not. D, E, F. Correlation between phase, resistance, and reactance values at $100 \mathrm{kHz}$ and age. Both phase and resistance show a significant relationship, whereas reactance does not.

Despite these results, a major issue remains: what is the actual clinical significance of these findings? Are the observed differences between LBP and healthy individuals, presumably reflecting differences in LPM health, actually contributing to the back pain or are they simply an associated, but unrelated effect? There is simply no way to tell from our results. Similarly, would correction or amelioration of these differences improve a given patient's discomfort? Although it is impossible to know from the data obtained here, such a question would be relatively simple to answer. For example, these measurements could be obtained before and after a dedicated program of core strengthening to determine if the values normalize with $a$ concomitant reduction in LBP.

Importantly, whereas we did identify significant differences between healthy individuals and those with low back pain of mixed etiology on a group basis, these differences were not such that we could use the test as a method for diagnosed LPM abnormalities in any one patient per se. There is considerable overlap and for any one individual it would not be possible to classify them accurately as healthy or with LBP based on a single impedance value. Our ROC analysis confirms an accuracy of only about $65 \%$ percent at best using this approach. However, this value is in keeping with standard clinical measures for radiculopathy alone [21,22].

Our correlation analyses did provide additional interesting information. First, it is clear that the reactance shows a strong relationship to age (see Figure $5 \mathrm{C}$ ) whereas the resistance does not. This fairly follows earlier work where age-related impedance changes were greater in reactance than for resistance [23]. Similar findings were also identified in a study assessing aged mice [24]. As for BMI, the resistance appears to be more strongly correlated, and is in keeping with our understanding of the impact of body fat on resistance, as compared to reactance, at $100 \mathrm{kHz}$ [25] and confirmed with previous and recent simulations [26-27]. Arguably, elevated body fat does indicate a predisposition to low back pain (e.g., perhaps due to the individual's being more sedentary). There is a strong association between body habitus and low back pain and thus these data may of interest from that perspective as well $[1,28]$.

Despite the potentially interesting results of this study, there are a number of important limitations. First, we did not perform a more extensive analysis of the underlying causes of LBP in this population. Similarly, we have provided no other quantification of severity of the back pain or its duration. Our main focus here was very much on looking for differences between groups as a first step in this direction and not attempting to use our measures as a surrogate of individual pain or disability. Third, we did not provide imaging or other quantitative indices of paraspinal muscle condition. This has been performed previously using computerized tomography [19]. Fourth, the EIM system, the mView, remains a relatively early version of EIM technology, and suffered from a number of limitations including noise and fairly rudimentary electrode array design. Newer versions of the technology, now in development, are likely to provide more robust and consistent data. Finally, as noted earlier, this data represents a single snap-shot in time and we cannot tell how these EIM values evolve over time or potentially may respond to therapy. 
In addition to these limitations, the focus of this paper has been in utilizing a single frequency $(100 \mathrm{kHz})$ in these measurements. Multifrequency data was obtained as well. Thus, future studies should incorporate these additional data as well as machine learning algorithms to fully utilize the very rich impedance data set being obtained.

\section{Conclusion}

In conclusion, this study provides proof-of-principle that EIM measurements, on a population basis, detect changes in muscle in patients with low back pain, and as a consequence, could serve as a rapid and convenient approach for quantifying LPM health going forward. Given the ongoing challenges in effectively managing and resolving low back pain, as well as the severity of the opioid epidemic in the United States, methods that can provide insight into back health, and that can potentially serve as indices of therapy response, may be of great value in the years to come.

\section{Acknowledgements \\ ZMT Zurich MedTech AG, Switzerland.}

\section{Conflict of interest}

Drs. Rutkove and Bohorquez hold equity in Myolex, Inc, have or currently serve on the board of directors, have received salary or consulting income from the company, and are named as inventors on patents owned or licensed to Myolex, Inc. Laura Freedman holds equity in Myolex and receives a salary. Martin Buck, receives a salary from Myolex. None of the other authors have any specific conflicts to report. This work was funded by National Institutes of Health Grant R44 AR064142 to Myolex, Inc (formerly Skulpt/Convergence Medical Devices).

\section{References}

1. Chen KY, Shaparin N, Gritsenko K. Low back pain. In: Pain Medicine: An Essential Review. 2017. Epub 1. Chen KY, Shaparin N, Gritsenko K. Low back pain. In: Pain Medicine: An Essential Review. 2017. Epub ahead of print 2017. https://doi.org/10.1007/978-3-319-43133-8_121

2. Maher C, Underwood M, Buchbinder R. Non-specific low back pain. The Lancet. Epub ahead of print 2017. https://doi.org/10.1016/s0140-6736(16)30970-9

3. Goubert D, Oosterwijck J Van, Meeus M, et al. Structural Changes of Lumbar Muscles in Non-Specific Low Back Pain. Pain Physician. 19(7), E985-E999.

4. Yamato TP, Maher CG, Saragiotto BT, et al. Pilates for low back pain. Sao Paulo Med J. Epub ahead of print 2016. https://doi.org/10.1590/1516-3180.20161344t1

5. Bogduk N. Management of chronic low back pain. Medical Journal of Australia, 180(2), 79-83.

6. Hayden JA, van Tulder MW, Malmivaara A, et al. Exercise therapy for treatment of non-specific low back pain. Cochrane database Syst Rev. Epub ahead of print 2005. https://doi.org/10.1002/14651858.cd000335.pub2
7. Chou R, Qaseem A, Snow V, et al. Diagnosis and treatment of low back pain: $A$ joint clinical practice guideline from the American College of Physicians and the American Pain Society. Annals of Internal Medicine. Epub ahead of print 2007. https://doi.org/10.7326/0003-4819-147-7-200710020-00006

8. Chang W-D, Lin H-Y, Lai P-T. Core strength training for patients with chronic low back pain. J Phys Ther Sci. Epub ahead of print 2015. https://doi.org/10.1589/jpts.27.619

9. Larivière $C$, Arsenault $A B$, Gravel $D$, et al. Surface electromyography assessment of back muscle intrinsic properties. J Electromyogr Kinesiol. Epub ahead of print 2003. https://doi.org/10.1016/s1050-6411(03)00039-7

10. Kamaz $M$, Kireşi $D$, Oğuz $H$, et al. CT measurement of trunk muscle areas in patients with chronic low back pain. Diagnostic Interv Radiol, 13(3), 144-148.

11. Kjaer P, Bendix T, Sorensen JS, et al. Are MRI-defined fat infiltrations in the multifidus muscles associated with low back pain? BMC Med. Epub ahead of print 2007. https://doi.org/10.1186/1741-7015-5-2

12. Lee H II, Song J, Lee HS, et al. Association between Crosssectional Areas of Lumbar Muscles on Magnetic Resonance Imaging and Chronicity of Low Back Pain. Ann Rehabil Med. Epub ahead of print 2012. https://doi.org/10.5535/arm.2011.35.6.852

13. Fortin $M$, Gibbons $L E$, Videman $T$, et al. Do variations in paraspinal muscle morphology and composition predict low back pain in men? Scand J Med Sci Sport. Epub ahead of print 2015. https://doi.org/10.1111/sms.12301

14. Sanchez B, Rutkove SB. Electrical Impedance Myography and Its Applications in Neuromuscular Disorders.

Neurotherapeutics 2017;14:107-18. https://doi.org/10.1007/s13311-016-0491-x

15. Shefner JM, Rutkove SB, Caress JB, et al. Assessing ALS progression with a dedicated electrical impedance myography system. Amyotroph Lateral Scler Frontotemporal Degener;(in press), 14(10), E0223265 https://doi.org/10.1080/21678421.2018.1510008

16. Rutkove SB, Kapur K, Zaidman CM, et al. Electrical impedance myography for assessment of Duchenne muscular dystrophy. Ann Neurol 2017;81:622-32. https://doi.org/10.1002/ana.24874

17. Li Z, Chen L, Zhu Y, et al. Handheld Electrical Impedance Myography Probe for Assessing Carpal Tunnel Syndrome. Ann Biomed Eng 2017;45:1572-80. https://doi.org/10.1007/s10439-017-1819-3

18. Tarulli AW, Duggal N, Esper GJ, et al. Electrical Impedance Myography in the Assessment of Disuse Atrophy. Arch Phys Med Rehabil;90. Epub ahead of print 2009. https://doi.org/10.1016/j.apmr.2009.04.007.

19. Zweig, M., \& Campbell, G. Receiver-Operating Characteristic (ROC) Plots- A Fundamental Evaluation Tool in Clinical Medicine. Clinical Chemistry, 39(4), 561-577.

20. Anderson DE, Bean JF, Holt NE, et al. Computed tomographybased muscle attenuation and electrical impedance myography as indicators of trunk muscle strength independent of muscle size in older adults. Am J Phys Med Rehabil 2014;93:553-61. https://doi.org/10.1097/phm.0000000000000059 
21. Iversen T, Solberg TK, Romner B, et al. Accuracy of physical examination for chronic lumbar radiculopathy. BMC Musculoskelet Disord. Epub ahead of print 2013. https://doi.org/10.1186/1471-2474-14-206

22. Ekedahl $H$, Jönsson $B$, Annertz $M$, et al. Accuracy of Clinical Tests in Detecting Disk Herniation and Nerve Root Compression in Subjects With Lumbar Radicular Symptoms. Arch Phys Med Rehabil. Epub ahead of print 2018. https://doi.org/10.1016/j.apmr.2017.11.006

23. Kortman HG, Wilder SC, Geisbush TR, et al. Age- and genderassociated differences in electrical impedance values of skeletal muscle. Physiol Meas 2013;34:1611-22. https://doi.org/10.1088/0967-3334/34/12/1611

24. Arnold WD, Taylor RS, Li J, et al. Electrical impedance myography detects age-related muscle change in mice. PLoS One;12. Epub ahead of print 2017.

https://doi.org/10.1371/journal.pone.0185614
25. Tarulli AW, Chin AB, Lee KS, et al. Impact of skin-subcutaneous fat layer thickness on electrical impedance myography measurements: An initial assessment. Clin Neurophysiol 2007;118:2393-7.

https://doi.org/10.1016/j.clinph.2007.07.016

26. Jafarpoor M, Li J, White JK, et al. Optimizing electrode configuration for electrical impedance measurements of muscle via the finite element method. IEEE Trans Biomed Eng 2013;60:1446-52. https://doi.org/10.1109/tbme.2012.2237030

27. Sim4Life and SEMCAD X Platforms. (n.d.). Retrieved from https://zmt.swiss/sim4life/. Visited: 12.12.2019.

28. Hoy D, Brooks P, Blyth F, et al. The Epidemiology of low back pain. Best Practice and Research: Clinical Rheumatology. Epub ahead of print 2010. DOI: 10.1016/j.berh.2010.10.002.ahead of print 2017.

https://doi.org/10.1007/978-3-319-43133-8_121. 